\title{
Calidad de vida en hijos de jornaleros agrícolas
}

Frida Itzel González Díaz ${ }^{1}$

\section{Aporte de Estudiante}

Posgrado

Resumen: La población infantil en algunas zonas rurales, se presenta como un sector con una potencial necesidad de realizarle estudios que contemplen la calidad de vida desde dimensiones subjetivas, debido a que son zonas consideradas como vulnerables por la alta precariedad y marginación, factores que pueden comprometer un óptimo desarrollo biopsicosocial de los niños. Abordar estudios desde una mirada cualitativa, permitirá no solo la visibilización de los niños como grupo social, sino que además aportará información desde su propia perspectiva de experimentar, interpretar y configurar el mundo que los rodea, de esta manera la información encontrada podrá ser de utilidad para fundamentar políticas públicas, programas y acciones para promover el bienestar y la calidad de vida. El siguiente escrito expone la problemática social y económica en la que se desenvuelven los niños hijos de jornaleros agrícolas, se hace una asociación de cómo estas condiciones desfavorables de carencia y marginación pueden incidir en el bienestar y la calidad de vida e influir a su vez, en la aparición de alteraciones psicológicas como la sintomatología depresiva. Se enmarca la importancia de profundizar en el estudio de la calidad de vida desde la metodología cualitativa, la cual sitúa al niño como un ser activo de la sociedad, que tiene la libertad de construir la realidad desde su mirada y con base en sus experiencias.

La región sur del estado de Jalisco, México, comprende en su mayoría municipios rurales dedicados a la agricultura, ganadería y actividades forestales. En las últimas dos décadas la agricultura de exportación se ha expandido de manera importante, lo que se traduce en un incremento de trabajadores del campo locales y migrantes, denominados jornaleros agrícolas. Esta mercantilización y producción agrícola, si bien genera empleo y ganancias monetarias, no se ven reflejadas en la mejora de la calidad de vida de la población, pues los índices de pobreza no han disminuido en los últimos 10 años (Escobar et al., 2020). Las condiciones de vida y laborales de estos trabajadores y sus familias, les impide en su mayoría acceder a una vida digna, acentuando la pobreza y la marginación social,

\footnotetext{
1 Estudiante de la Maestría en Psicología con Orientación en Calidad de Vida y Salud, CUSUR, Universidad de Guadalajara. Correo electrónico: fri.dha@hotmail.com
} 
convirtiéndolos a ellos y a sus familias en el grupo social más desvalido pobre y vulnerable del país (Andrade, 2013).

La actual pandemia del COVID-19 ha vulnerado aún más a este grupo, el cual, al formar parte esencial de la producción de alimentos, no ha suspendido sus labores, acrecentando la posibilidad de contagiarse y enfermar (La Conferencia Interamericana de Seguridad Social, [CISS] 2020). Además, la modificación de las clases presenciales a modalidad en línea genera preocupación a los padres que dejan sin supervisión a los niños gran parte del día, la ausencia de apoyo de un adulto en clases puede dificultar la realización de las tareas escolares, aunado a que las condiciones de vivienda son inadecuadas para estudiar y que en algunos casos no se cuenta con los servicios y equipo básico para realizar las tareas, estos factores propician el rezago educativo y posibilitan alteraciones en la salud mental y bienestar (Zenteno, 2020).

Las circunstancias de pobreza y marginación de las que son parte las familias jornaleras, incrementan la vulnerabilidad psicológica lo que puede traer consigo dificultades que pueden influir de manera negativa en el desarrollo afectivo de los hijos, ello, hace más probable la aparición de alteraciones psicológicas como la depresión (Greco, et al., 2007; Kotliarenco, et al., 1997) considerada uno de los trastornos más recurrentes en la niñez (Rooney, et al., 2013). Los síntomas depresivos, aunque no alcancen un nivel de diagnóstico del trastorno podrán incidir negativamente en las áreas del desarrollo cognitivo, emocional, somático y comportamental (Garaigordobil, et al., 2019) además de que se asocia con menor bienestar y calidad de vida en los niños (Martinsen, et al., 2016). Por lo anterior, la evaluación, detección y tratamiento temprano, son relevantes para brindar alternativas que contribuyan a la promoción del bienestar de la niñez.

La calidad de vida en la niñez, puede ser definida como una percepción subjetiva de bienestar y satisfacción de acuerdo a la propia experiencia, evaluación que se realiza de los dominios de la vida social, física y psicológica Mattejat y Remschmidt (1998) (citado en Martinsen, et al., 2016). Es un constructo que se entenderá de manera diferente de acuerdo al grupo social, a la zona geográfica, a la cultura y sobre todo variará de significado con base a la medición que el profesional utilizará para dar respuesta a lo que quiere identificar, es esa subjetividad la que lo vuelve un concepto difícil de medir (Sanabria, 2016).

La niñez se posiciona como la base para la construcción y la promoción de la calidad de vida, en la que es importante comprender aquellas dificultades y expectativas, los niños al igual que los adultos forman parte trascendental de la estructura social (Mieles y Acosta, 2012). La mayoría de los estudios han abordado la calidad de vida contemplando indicadores de salud en áreas hospitalarias, siendo menor la evidencia que se tiene de investigaciones en poblaciones rurales desde dimensiones subjetivas. Algunas investigaciones comparativas 
entre niños rurales y urbanos (Solano, et al., 2017; Urzúa y Caqueo-Urízar 2013) sugieren que las circunstancias de carencia en recursos materiales o servicios no serán una condicionante para presentar una menor o mayor calidad de vida, sino que son las experiencias de violencia o las interacciones sociales las principalmente asociadas.

De lo anterior surge la importancia de integrar al método cualitativo en el estudio de la calidad de vida, el cual permite indagar sobre aspectos subjetivos basados en la experiencia de la persona y en la libertad de interpretar el mundo que les rodea (Tonon, 2010). Una técnica básica en esta metodología es la entrevista semi-estructurada, ese intercambio de ideas entre dos o más personas, la cual requiere de un esfuerzo escrupuloso de comunicación y alto nivel analítico, donde se van entretejiendo y reconstruyendo realidades, este acercamiento exige también un trabajo introspectivo del propio investigador (Tonon, 2009).

Con dicha perspectiva se pretende explorar y comprender fenómenos que acontecen en las cotidianidades de los hijos de jornaleros agrícolas locales del sur de Jalisco en México, se plantean estudios que permitan identificar cómo experimentan y significan estos niños sus interacciones familiares, qué tipo de expresividad emocional y afectiva contribuye al bienestar o por el contrario, descubrir qué condiciones familiares, sociales o materiales perciben, pueden propiciar alteraciones psicológicas o disminución en su calidad de vida. Asimismo, comprender cómo el niño configura la realidad que le rodea, qué aspectos de su entorno pueden ser para él la fuente de motivación o de dificultades. Esta metodología brinda la pauta para saber qué es lo que hace que un niño inmerso en circunstancias de dificultad logre construir su bienestar y otro no.

Pueden existir diversos planteamientos que motiven a la investigación en este grupo y conforme se explore dicha realidad irán surgiendo nuevas dudas con las que se vaya armando un rompecabezas respecto a la experiencia de ser niña o niño de comunidades en las que existen carencias estructurales, falta de oportunidades y donde la fuente principal de trabajo es la agroindustria, la cual se presenta como la única oportunidad para poder acceder a una vida más digna.

\section{Conclusión}

Llevar a cabo estudios con personas circunscritas en realidades socioeconómicas adversas desde una perspectiva fenomenológica permitirá comprender de qué manera la precariedad, el trabajo, la migración o la violencia tienen una implicación en la salud mental y el bienestar. Al mismo tiempo, brindarán la oportunidad de reflexionar sobre nuevas formas de significar los conceptos de bienestar, calidad de vida y felicidad. 
El acercamiento a estos grupos sociales contribuirá también a la visibilización, las conclusiones que se generen son una oportunidad para contribuir a la formulación de programas y acciones en beneficio de esta población, donde hasta el momento las políticas públicas gubernamentales han resultado ineficaces para disminuir la brecha de desigualdad.

La autora del presente escrito agradece al CONACyT por la beca nacional 845004 otorgada durante los estudios de maestría.

\section{Referencias}

Andrade, K. (2013). Excluidos y estigmatizados los jornaleros migratorios en Tamaulipas. Editorial Miguel Ángel Porrúa.

Conferencia Interamericana De Seguridad Social (CISS) (2020). Las y los jornaleros agrícolas ante el Covid-19. CISS website https://ciss-bienestar.org/wpcontent/uploads/2020/05/las-y-los-trabajadores-jornaleros-agricolas-ante-elcovid19.pdf

Escobar, A., Martínez, E. y Judd M. (2020). Agricultura de exportación y pobreza en el Valle de Ciudad Guzmán. CIESAS Boletín 2.

Garaigordobil, M., Bernaras E. y Jaureguizar J. (2019). Evaluación de un programa universal de prevención de la depresión en niños y niñas de educación primaria. Revista de psicología y educación, 14(2), 87-89. https://doi.org/10.23923/rpye2019.01.174

Greco, C., Morelato, G. e Ison M. (2007). Emociones positivas: Una herramienta psicológica para promocionar el proceso de resiliencia infantil. Psicodebate.Psicología, Cultura y Sociedad, 7, 81-94.

Kotliarenco, M., Cáceres, I., y Fontecilla, M. (1997). Estado de arte en Resiliencia. Organización Panamericana de la Salud. PAHO website: https://www.paho.org/Spanish/HPP/HPF/ADOL/Resil6x9.pdf

Martinsen, K., Neumer, S., Holen, S., Waaktaar, T., Sund, A., y Kendall, P. (2016). Selfreported quality of life and self-esteem in sad and anxious school children. BMC Psychology, 4(45). https://doi.org/10.1186/s40359-016-0153-0

Mieles, M. y Acosta, A. (2012). Calidad de vida y derechos de la infancia: un desafío presente. Revista Latinoamericana de Ciencias Sociales, Niñez y Juventud, 10 (1), 205-217. 
Rooney, R., Hassan, S., Kane, R., Roberts, C. M. y Nesa, M. (2013). Reducing depression in 9-10 year old children in low SES schools: A longitudinal universal randomized controlled trial. Behaviour Research and Therapy, 51, 845-854. https://doi.org/10.1016/j.brat.2013.09.005

Sanabria, M. (2016). Calidad de vida e instrumentos de medición (Tesis de pregrado). Universidad de la Laguna, España.

Solano, F., Vilela-Estrada, M., Meza-Liviapoma, J., Araujo-Chumacero, M., Vilela-Estrada, A. y Mejia C. (2017). Factores sociofamiliares asociados a la calidad de vida en niños de colegios de la ciudad de Piura, Perú. Revista Chilena de Pediatría, 88(2), 223-229. http://dx.doi.org/10.1016/j.rchipe.2016.07.012

Tonon, G., (2009). La entrevista semi-estructurada como técnica de investigación. En G. Tonon (Ed.), Reflexiones latinoamericanas sobre investigación cualitativa (págs. 47-68). Prometeo Libros-UNLAM.

Tonon, G., (2010). Los estudios sobre calidad de vida y la perspectiva de la psicología positiva. Psicodebate, Psicología, Cultura y Sociedad, 10, 73-8.

Urzúa, A. y Caqueo-Urízar, A. (2012). Calidad de vida: Una revisión teórica del concepto. Terapia Psicológica, 30(1), 61-71.

Zenteno, R. (20 de abril de 2020). Who takes care of the farmworkers' children? Facing South. Facing South website: https://www.facingsouth.org/2020/04/who-takescare-farmworkerschildren?fbclid=IwAR2I7f_pDU2F3Y_rypbTb5wLMskqMGOG06Ad9c9chZtSGdq6 pKhl70psPww 